\title{
Characterization of the Novel Insecticidal Crystal Protein Cyt3Aa1 from Bacillus thuringiensis TD516
}

\author{
Jun Zhu1,2*, Zizhong Zhu1 ${ }^{*}$, Baoli Zhang1, Xu Liu1, Yiping Liu1, Aiping Zheng, ${ }^{1,2}$, Shiquan Wang1, \\ Shuangcheng Li1 ${ }^{1}$,iming Deng1, Huainian Liu' ${ }^{1}$, Yueyang Liang ${ }^{1}$, Ting Zou' ${ }^{1}$, Aijun Wang1, \\ Lingxia Wang ${ }^{1}$, Ping Li ${ }^{1,2 \#}$
}

${ }^{1}$ Rice Research Institute of Sichuan Agricultural University, Chengdu, China

${ }^{2}$ State Key Laboratory of Crop Gene Exploration and Utilization in Southwest China, Chengdu, China

Email: "liping6575@163.com

How to cite this paper: Zhu, J., Zhu, Z.Z., Zhang, B.L., Liu, X., Liu, Y.P., Zheng, A.P., Wang, S.Q., Li, S.C., Deng, Q.M., Liu, H.N., Liang, Y.Y., Zou, T., Wang, A.J., Wang, L.X. and Li, P. (2020) Characterization of the Novel Insecticidal Crystal Protein Cyt3Aa1 from Bacillus thuringiensis TD516. Advances in Microbiology, 10, 349-358. https://doi.org/10.4236/aim.2020.107025

Received: July 7, 2020

Accepted: July 21, 2020

Published: July 24, 2020

Copyright $\odot 2020$ by author(s) and Scientific Research Publishing Inc. This work is licensed under the Creative Commons Attribution International License (CC BY 4.0).

http://creativecommons.org/licenses/by/4.0/

\begin{abstract}
Bacillus thuringiensis $(\mathrm{Bt})$ produces two families of insecticidal crystal proteins, i.e., crystalline (Cry) and cytolytic (Cyt) toxins. Cyt3Aa1, the newest Cyt family member, is produced by Bt TD516. Bioassay results have shown that Cyt3Aa1 has weak hemolytic activity against human red blood cells and is not toxic to $A$. aegypti larvae, but causing a teratogenic effect. The three-dimensional structure of Cyt3Aal has a typical cytolysin fold containing a $\beta$-sheet held by two surrounding $\alpha$-helical layers, resembling the previously reported Cyt1Aa and Cyt2Aa structures, which indicated that Cyt3Aa1 might be a membrane-perforation toxin and could induce synergism with Cry protein. This study provides a new source of insecticidal crystal proteins, and presents a foundation for understanding the biological characterization of it, which will aid in the development of strategies to cope with the potential problem of insect resistance.
\end{abstract}

\section{Keywords}

TD516, Cyt3Aa1, Activity Assays, Three-Dimensional Structure

\section{Introduction}

During sporulation, Bt produces two families of insecticidal crystal proteins, Cry and Cyt. To date, 78 families of Cry toxins had been obtained, although only two families of Cyt toxins (Cyt1 and Cyt2) had been obtained (http://www.lifesci.sussex.ac.uk/home/Neil Crickmore/Bt/).

${ }^{\star} J u n$ Zhu, and Zizhong Zhu contributed equally to this work. 
The Cry and Cyt toxins have different modes of action of against insects [1] [2], the Cry toxins bind to specific protein receptors of insects, and Cyt toxins act non-specifically by direct interaction with membrane lipids of insects [3] [4]. The Cyt proteins mainly have activity against larvae of Diptera, Hemiptera, and Aphidinae [5] [6] [7]. Additionally, the Cyt1Aa and Cyt2Aa can synergize Cry and Vip toxicity against mosquitoes, respectively [7] [8] [9], and Cyt toxin may ease the threat of insect resistance [10]. The crystal structures of Cyt1Aa and Cyt2Aa have been determined by X-ray crystallography, and both have a single $\alpha / \beta$ domain composed of two outer layers of $\alpha$-helix hairpins wrapped around a $\beta$-sheet [2] [3]. The analysis of the structural characterization of Cyt protein could bridge the structure-knowledge gap.

\section{Materials and Methods}

\subsection{Sequence Analysis of Cyt3Aa1}

The sequence homology of Cyt3Aal was determined using the NCBI protein-protein BLAST program (http://www.ncbi.nlm.nih.gov/BLAST). The alignment of the Cyt3Aa1, Cyt1Aa1 and Cyt2Aal sequences was performed using Clustal W software (European Bioinformatics Institute, http://www.ebi.ac.uk/clustalw/). Phylogenetic analysis of the Cyt proteins was performed using MEGA 6.0 software [11].

\subsection{Insecticidal and Hemolytic Activity Assays of Cyt3Aa1}

The cyt3Aal gene was ligated into the cloning vector pGEM-T (Promega), which was digested with $K p n I$ and $S a l I$ and inserted into the same sites in the pET32a expression vector (Novagen) to obtain a recombinant plasmid (pET-Cyt3A). Subsequently, pET-Cyt3A was transformed into E. coli Arctic Express cells (Zoon Bio). The expression of the cyt gene was assessed as described by Cohen et al. [2]. The protein preparation was analyzed by SDS-PAGE as described by Green et al. [12].

The activity of Cyt3Aal was tested against larvae of $A$. aegypti (Diptera) as previously described [13]. The hemolytic activity of the Cyt3Aa1 protein was determined using human red blood cells as described by Ragni et al. and Juárez-Pérez et al. [14] [15]. Protein was added at six different concentrations $(0.1$ to $50 \mu \mathrm{g} / \mathrm{ml})$, and the hemolytic rate (\%) was examined after $4 \mathrm{~h}$. Each bioassay was repeated at least five times, the $50 \%$ lethal concentrations were determined by Probit analysis, and the hemolytic rate of Cyt3Aa1 was estimated as follows: hemolytic rate $(\%)=($ supernatant $\mathrm{Hb} *[1-\mathrm{Hct} \%]) /$ total $\mathrm{Hb}{ }^{*} 100$.

\subsection{Construction of a Three-Dimensional Structure Model of Cyt3Aa1 by Homologous Modeling}

An alignment of Cyt3Aa1 and Cyt2Aa1 was produced using the Clustal W program, and the alignment was submitted to Swiss-Model (http://www.expasy.ch/spdbv/). The preliminary model of Cyt3Aa1 was predicted using the server SWISS-MODEL 
(http://swissmodel.expasy.org/) [16].

The minimized model structure of Cyt3Aal was then determined to perform energy minimization using the PMEMD module of the AMBER 14.0 software to eliminate unreasonable contacts. Additionally, the minimized model was assessed and validated using the PROCHECK validation server for molecular dynamics simulations [17]. Using the structure of Cyt3Aal lacking unfavorable contacts, the entire system was gradually heated to $310 \mathrm{~K}$ using Langevin dynamics. A 10- $\AA$ cutoff was used for the non-bonded interactions. The Particle Mesh Ewald (PME) method was used to treat long-range electrostatic interactions. In addition, the ProSA program (https://prosa.services.came.sbg.ac.at/prosa.php) was used to evaluate the computational model.

\section{Results and Discussion}

\subsection{Sequence Analysis and Biological Activity of Cyt3Aa1}

The gene cyt3Aal (GenBank accession: HM596591) cloned from BtTD516 encoded a 259 -amino acid protein with a molecular mass of $29.5 \mathrm{kDa}$. The currently known subfamily members of Cyt2-type and Cyt3Aal share a high level of sequence identity (Figure 1), the figure of which was created using ESPript [18], and Cyt3Aal has a maximum $39.04 \%$ amino acid sequence homology with Cyt2Aa.

Phylogenetic analysis of Cyt proteins was conducted using MEGA 6.0 [11]. Most of the known Cyt proteins are specific against the larvae of Diptera insects,
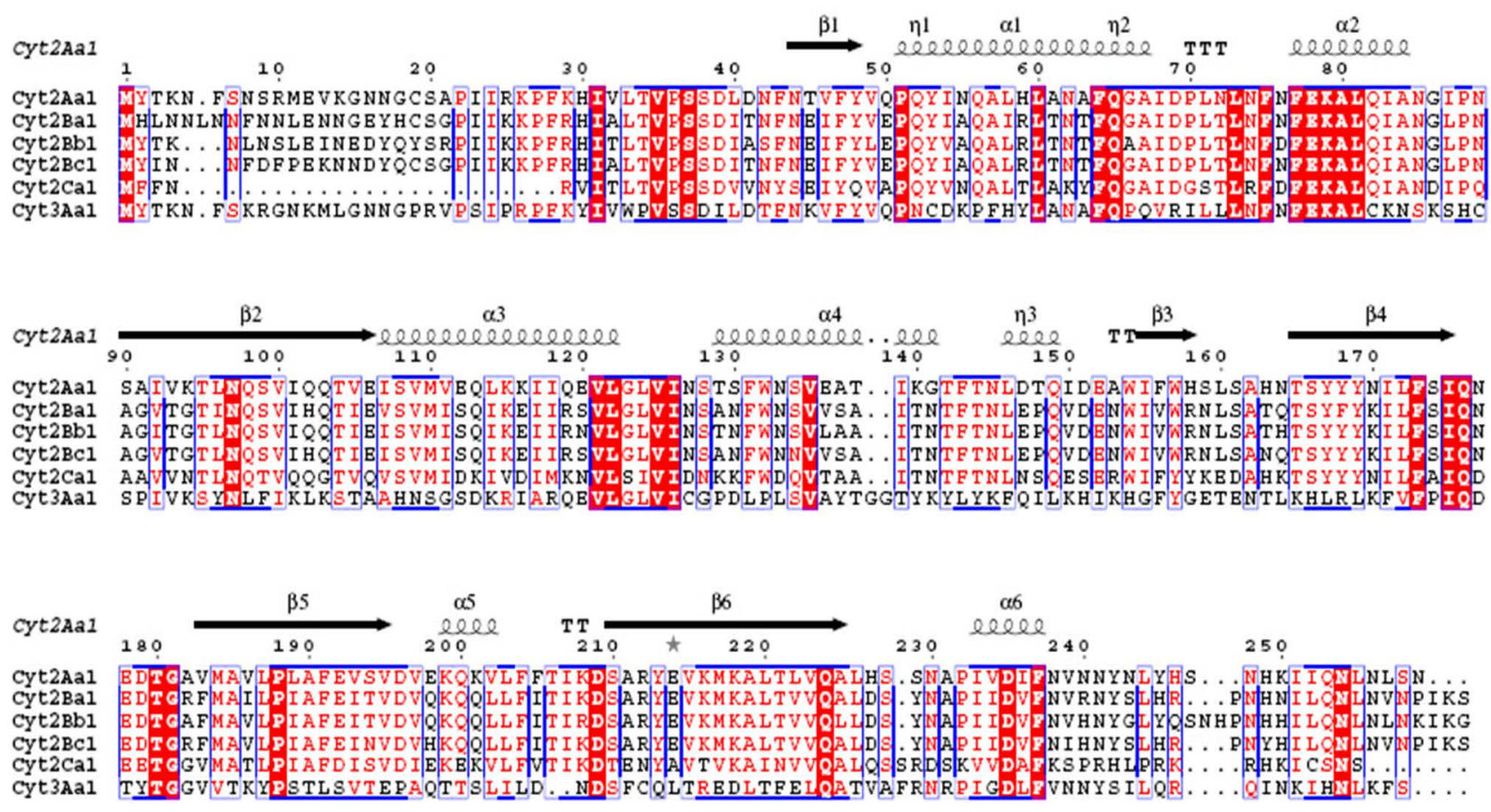

Figure 1. Sequence alignment of Cyt family. $\alpha$-Helices and $\eta$-helices are depicted using spirals, and $\beta$-strands are indicated by arrows. The residues conserved in all 6 proteins are shown in red blocks, and the residues shown in red text are identical in at least 4 of the 6 proteins. 
such as Cyt1A, Cyt2B, and Cyt2Aa [2] [7] [8]. The phylogenetic analysis of the three families of Cyt proteins showed that they may have evolved from a common ancestor (Figure 2), suggesting that Cyt3Aal may have potential mosquitocidal activity.

The SDS-PAGE results showed that cry $3 A a 1$ was highly expressed in E. coli as inclusion bodies (47-kDa protein as well as the approximately $19-\mathrm{kDa}$ Trx-tagin pET32a) (Figure 3). The approximately 30-kDa protein purified (Cyt3Aa1) was used to assay the biological activity against $A$. aegypti and human red blood cells. The bioassay results showed that Cyt3Aal was not toxic to $A$. aegypti, but causing a teratogenic effect, which appears to be a new way for $A$. aegypti control. In contrast to Cyt1Aa and Cyt2Ba, the hemolytic activity of Cyt3Aa1 was very weak $(0.0024 \%-0.034 \%)$ when it was solubilized, a finding that was similar

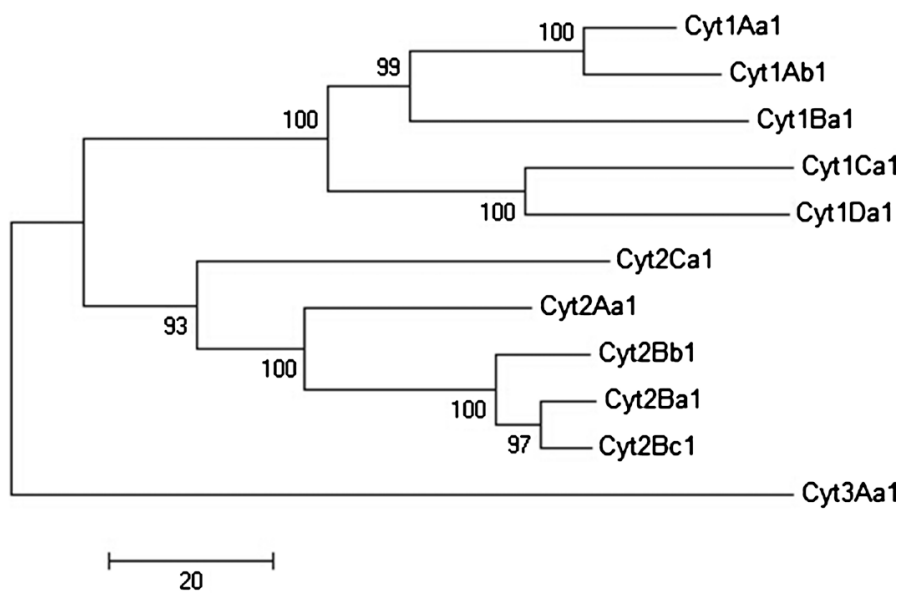

Figure 2. Phylogenetic analysis of Cyt. The numbers at the nodes represent the percentages of bootstrap resamplings based on 500 replicates.

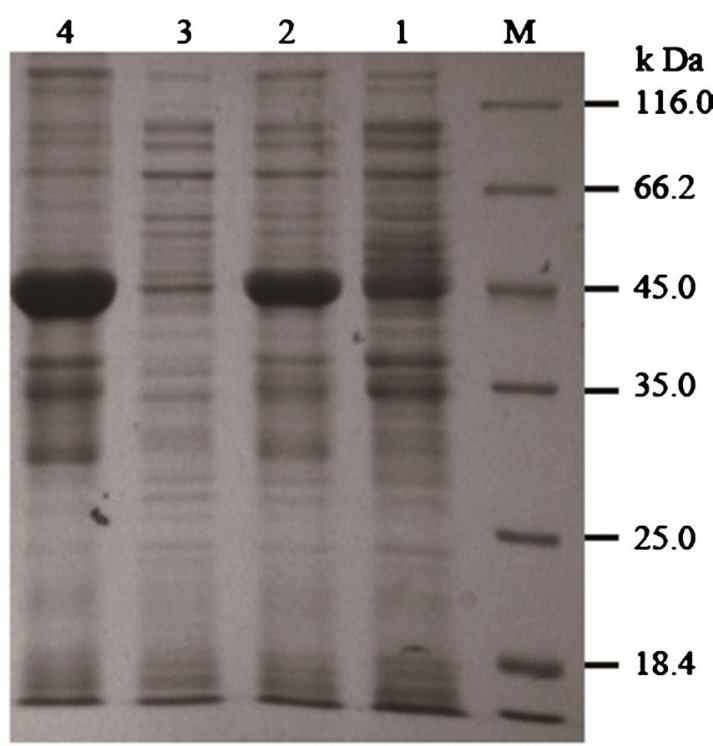

Figure 3. SDS-PAGE analysis of the expression forms of Cyt3Aa1. Lane M: Protein Marker; Lane 1: un-induced; Lane 2: induced; Lane 3: supernatant of induction; Lane 4: precipitate of induction. 
to the hemolytic activity of Cyt2Bc2 [15].

\subsection{The Three-Dimensional of Cyt3Aa1 by Homology Modeling}

In the absence of an experimentally determined structure, computational homology modeling has an added advantage due to the use of template information from known biological samples [19]. In this study, 1CBY (PDB Code of Cyt2Aa1) was employed to be the most suitable template for homology modeling of Cyt3Aa1, which has $43.13 \%$ similarity to Cyt3Aa1 [20], and the structural models of the Cyt3Aa1 toxic core were obtained comprising 223 of the 259 amino acid primary structure.

The minimized model structure of Cyt3Aal constructed by the PMEMD module of AMBER 14.0 was assessed and validated using the PROCHECK [17]. The result showed that all the residues were found in the allowed region (Figure 4),

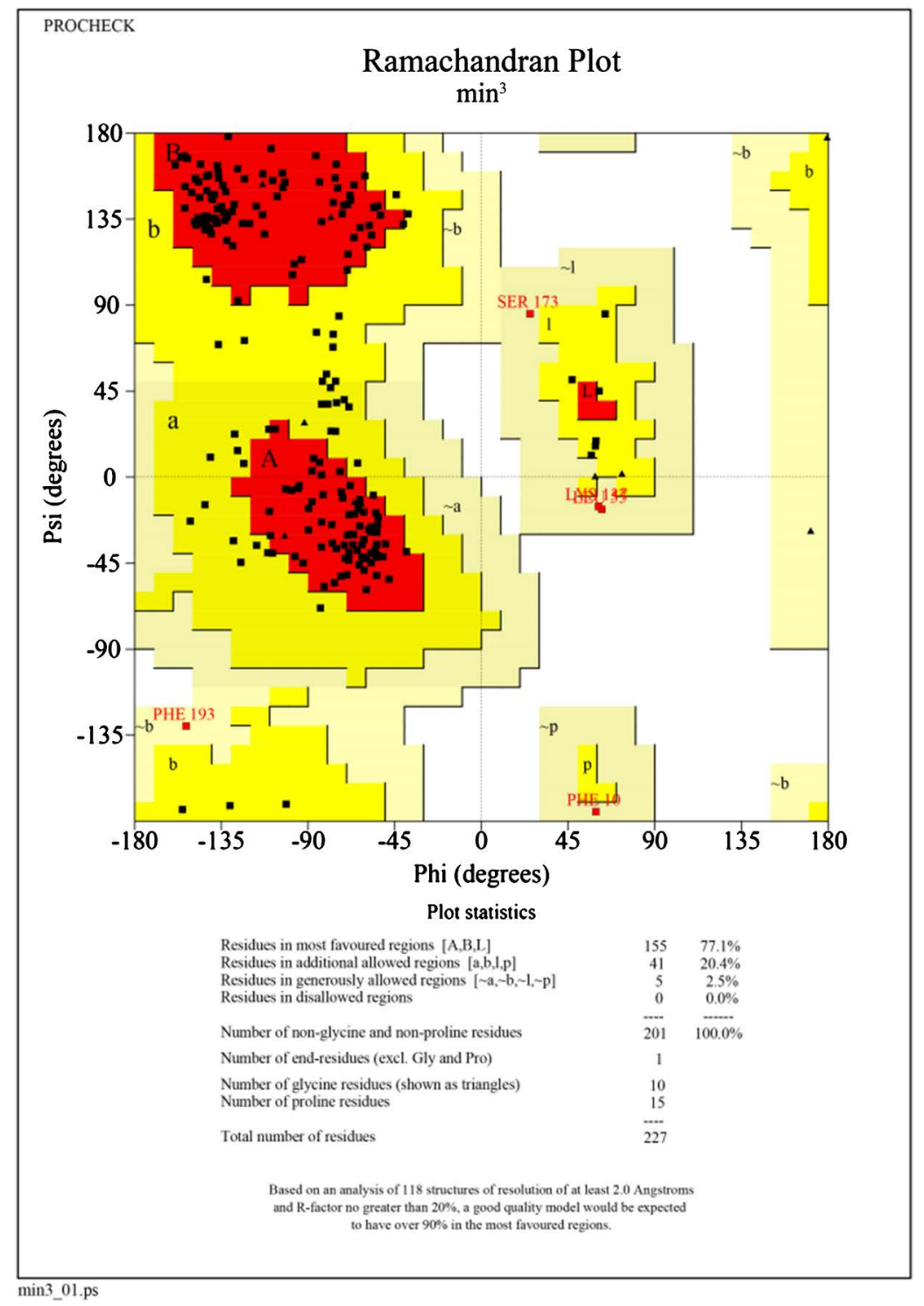

Figure 4. Ramachandran plot of Cyt3Aa1. 
which indicated that the Cyt3Aa1 model was of good quality and considered fit for molecular dynamics simulations. Subsequently, the model was optimized by the force field FF99SB module of AMBER 14.0. Then the final frame structure of Cyt3Aa1 was simulated and generated by PyMOL (http://www.pymol.org/) (Figure 5). The most covalent/non-covalent bonds, bond angles, and torsion angles of the structure have the expected values for a naturally folded protein assessed by Protein Structure Evaluation Suite and Server

(http://www.prosess.ca/index.php). Additionally, the $z$-score $(-2.82)$ of the model was within the range of similar protein model derived by NMR and X-ray crystallography evaluated using ProSA program

(https://prosa.services.came.sbg.ac.at/prosa.php) [21], indicating that the model was in the native conformation (Figure 6). All these results indicated that the generated model was valid with good stereochemical quality.

\subsection{Characterization of the Three-Dimensional of Cyt3Aa1}

The structure of Cyt3Aal resembles the crystal structure of Cyt2Aa1 [3], which also has a single $\alpha / \beta$ domain (Figure 5). The $\beta$-sheet of Cyt3Aal consists of four main $\beta$-strands arranged in space as $\beta 2 \downarrow, \beta 6 \uparrow, \beta 5 \downarrow$, and $\beta 4 \uparrow$ and was flanked by two $\alpha$-helical layers ( $\alpha 2-\alpha 3$ on one side, and $\alpha 1-\alpha 4$ on the other). The mechanism of action of Cyt1Aa1, Cyt2Ba1, Cyt2Aa1, and VVA2 (PDB code:

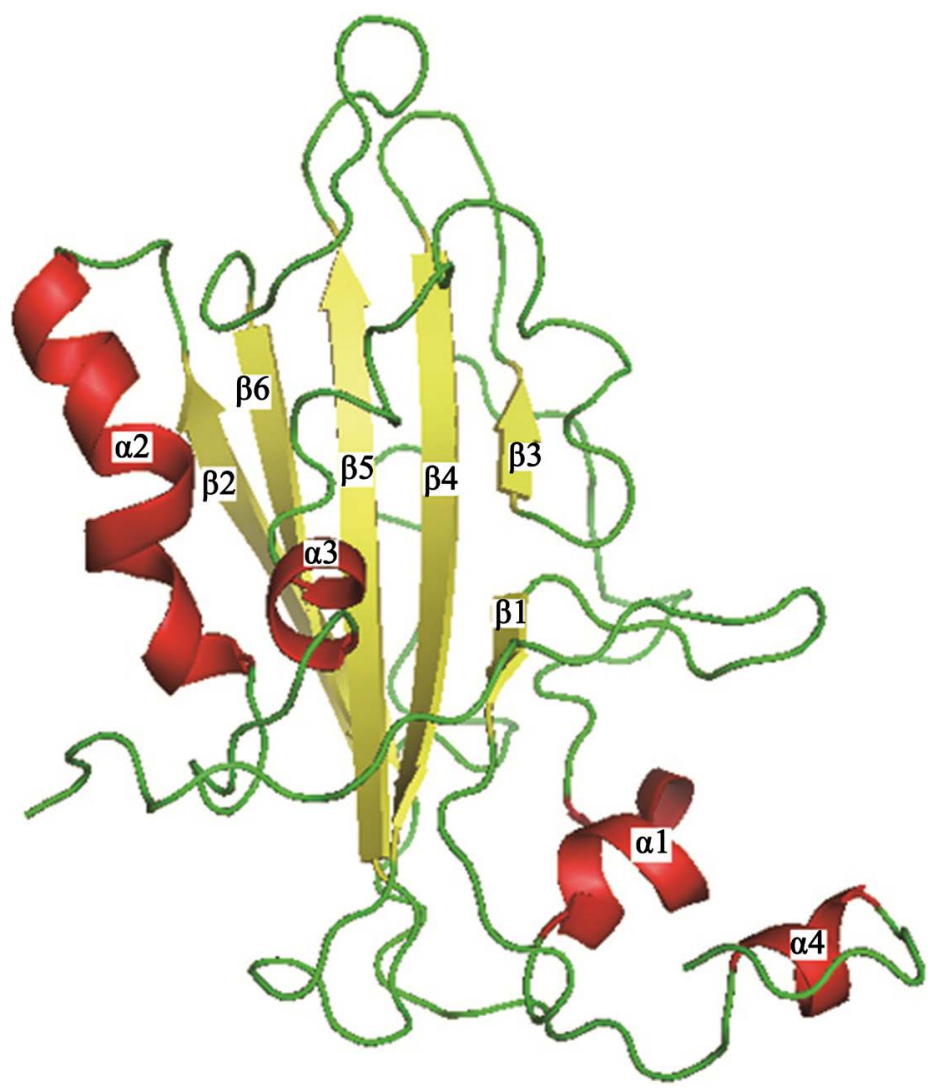

Figure 5. Ribbon representations of Cyt3Aa1. $\alpha$-Helices are shown in red, $\beta$-sheets are shown in yellow. 


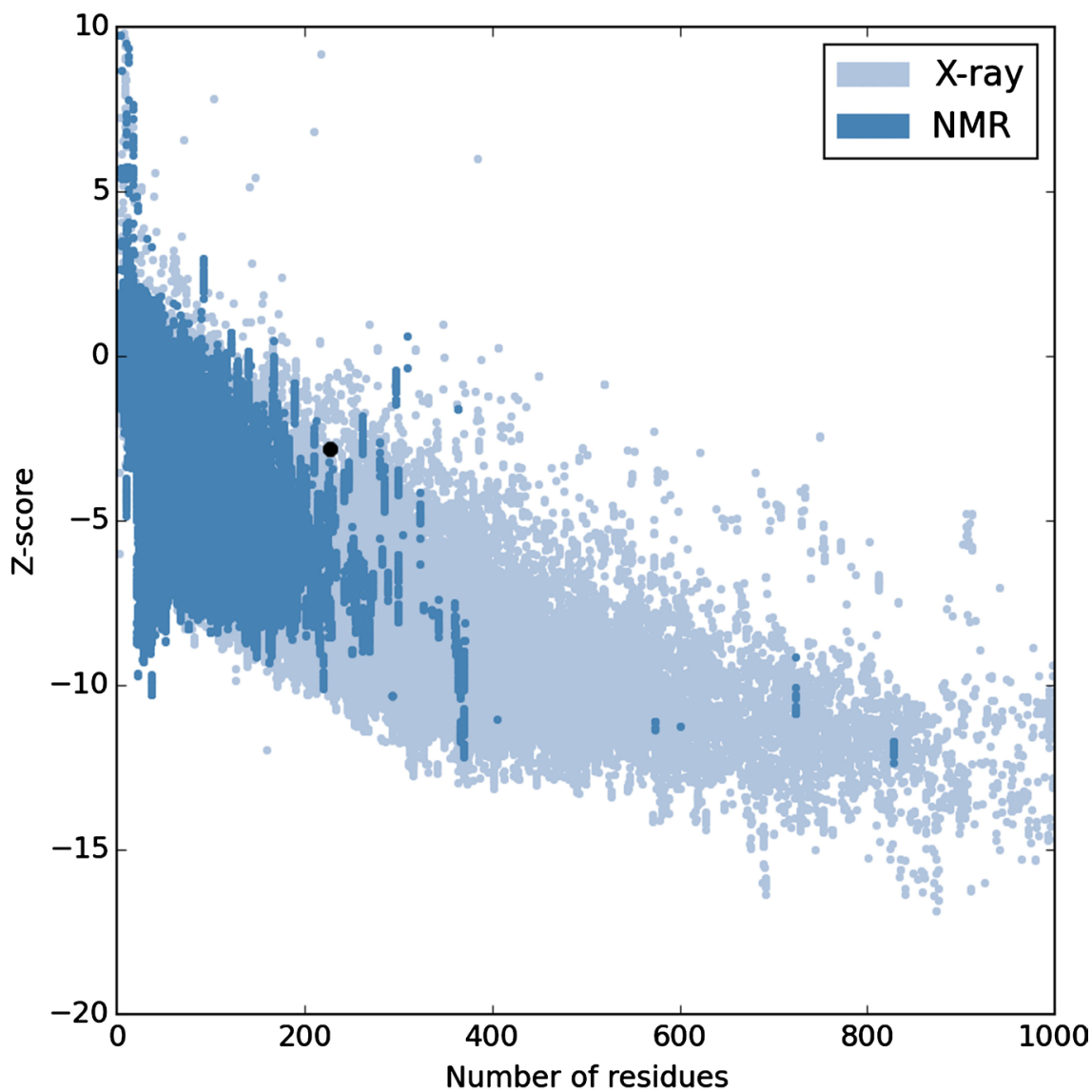

Figure 6. Evaluation of the Cyt3Aa1 model. The $z$-scores of all the protein chains in PDB were determined by X-ray crystallography (light blue) and NMR spectroscopy (dark blue) with respect to their length. The $z$-scores are indicated as large black dots. The plot shows only chains with $<1000$ residues and a $z$-score $\leq 10$.

$1 \mathrm{VCY})$ is thought to be that the two surrounding $\alpha$-helical layers swing away from the $\beta$-sheet upon membrane contact to expose the hydrophobic $\beta$-sheet, which can insert into the membrane to form a $\beta$-barrel pore, resulting in colloid osmotic lysis [22] [23]. The Cyt3Aal also has two $\alpha$-helical layers with considerable variation that surround the $\beta$-sheets, and all $\beta$-sheets adopt a cytolysin fold, which revealed that Cyt3Aa1 toxin might be a membrane-perforation toxin.

Cyt1Aa could synergize the activities of Cry11Aa to A.aegypti larvae [24]. The three single residues (K198, E204, and K225) of two binding epitopes $\left({ }_{196} \mathrm{EIKVSAVKE}_{204}\right.$ and ${ }_{220} \mathrm{NIQSLKFAQ}_{228}$ ) of Cyt1Aa were shown to be mainly involved in the interaction between Cyt1Aa1 and Cry11Aa, Cry4Ba, respectively [25] [26]. Interestingly, the three single residues are charged in most of the Cyt family members, and the corresponding residues of Cyt3Aa1 (P198, L204, and A225) are also charged. Mapping of the three charged residues on the Cyt3Aal structure revealed that the residues are exposed to the surface of the toxin (Figure 7), which might introduce binding sites and induce synergism with Cry protein. 


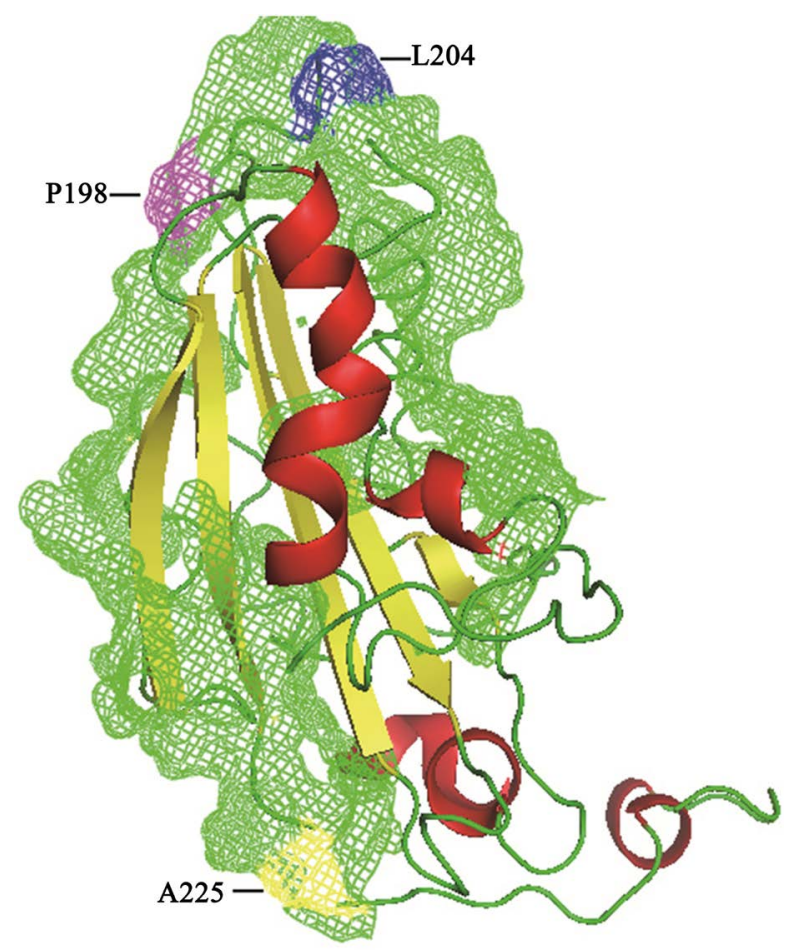

Figure 7. Mapping of the three charged residues on the Cyt3Aal model.

\section{Conclusion}

In this study, we report the biological characterization and the structure of Cyt3Aa1, which not only provide foundation for understanding the mechanism underlying its toxicity, but will also help in the design of improved membrane-active toxins with novel specificities.

\section{Authors' Contributions}

The experiments and the writing of the manuscript were performed by Jun Zhu, Zizhong Zhu and Baoli Zhang. The sequence analysis and the editing of the manuscript were performed by Yiping Liu, Xu Liu, Shiquan Wang, Shuangcheng Li. The construction of the model of Cyt3Aal by homologous modeling was performed by Qiming Deng, Huainian Liu, Yueyang Liang. And the insecticidal and hemolytic activity assays of Cyt3Aa1 were performed by Lingxia Wang, Ting Zou, and Aijun Wang. The design and support of all the manuscript were performed by Aiping Zheng, and Ping Li. All authors read and approved the final manuscript.

\section{Acknowledgments}

This study was supported by Sichuan Science and Technology Program 2017JY0120, 2018NFP0020, 20ZDYF1833, 2020YFN0006; National key research and development projects 2017YFD0100201.

\section{Conflicts of Interest}

The authors declare no conflicts of interest regarding the publication of this paper. 


\section{References}

[1] Bravo, A., Gill, S.S. and Soberón, M. (2007) Mode of Action of Bacillus thuringiensis Cry and Cyt Toxins and Their Potential for Insect Control. Toxicon, 49, 423-435. https://doi.org/10.1016/j.toxicon.2006.11.022

[2] Cohen, S., Albeck, S., Ben-Dov, E., et al. (2011) Cyt1Aa Toxin: Crystal Structure Reveals Implications for Its Membrane-Perforating Function. Journal of Molecular Biology, 413, 804-814. https://doi.org/10.1016/j.jmb.2011.09.021

[3] Li, J., Koni, P.A. and Ellar, D.J. (1996) Structure of the Mosquitocidal $\delta$-Endotoxin CytB from Bacillus thuringiensissp. Kyushuensis and Implications for Membrane Pore Formation. Journal of Molecular Biology, 257, 129-152. https://doi.org/10.1006/jmbi.1996.0152

[4] Promdonkoy, B. and Ellar, D. (2003) Investigation of the Pore-Forming Mechanism of a Cytolytic $\delta$-Endotoxin from Bacillus thuringiensis. Biochemical Journal, 374, 255-259. https://doi.org/10.1042/bj20030437

[5] Delécluse, A., Juárez-Pérez, V. and Berry, C. (2000) Vector-Active Toxins: Structure and Diversity. In: Charles, J.F., Delécluse, A. and Roux, C.N.L., Eds., Entomopathogenic Bacteria: From Laboratory to Field Application, Kluwer Academic Publishers, Boston, 101-125. https://doi.org/10.1007/978-94-017-1429-7_6

[6] Chougule, N.P., Li, H., Liu, S., et al. (2013) Retargeting of the Bacillus thuringiensis Toxin Cyt2Aa against Hemipteran Insect Pests. Proceedings of the National Academy of Sciences of the United States of America, 110, 8465-8470. https://doi.org/10.1073/pnas.1222144110

[7] Yu, X., Tao, L., Sun, Z., et al. (2012) Co-Expression and Synergism Analysis of Vip3Aa29 and Cyt2Aa3 Insecticidal Proteins from Bacillus thuringiensis. Current Microbiology, 64, 326-331. https://doi.org/10.1007/s00284-011-0070-7

[8] Berry, C., O’Neil, S., Ben-Dov, E., et al. (2002) Complete Sequence and Organization of pBtoxis, the Toxin-Coding Plasmid of Bacillus thuringiensis Subsp. Israelensis. Applied and Environmental Microbiology, 68, 5082-5095. https://doi.org/10.1128/AEM.68.10.5082-5095.2002

[9] Pardo-López, L., Gómez, I., Rausell, C., et al. (2006) Structural Changes of the CrylAc Oligomeric Pre-Pore from Bacillus thuringiensis Induced by $\mathrm{N}$-acetylgalactosamine Facilitates Toxin Membrane Insertion. Biochemistry, 45, 10329-10336. https://doi.org/10.1021/bi060297z

[10] Ferré, J. and Van Rie, J. (2002) Biochemistry and Genetics of Insect Resistance to Bacillus thuringiensis. Annual Review of Entomology, 47, 501-533. https://doi.org/10.1146/annurev.ento.47.091201.145234

[11] Tamura, K., Stecher, G., Peterson, D., et al. (2013) MEGA6: Molecular Evolutionary Genetics Analysis Version 6.0. Molecular Biology and Evolution, 30, 2725-2729. https://doi.org/10.1093/molbev/mst197

[12] Green, M.R. and Sambrook, J. (2012) Molecular Cloning: A Laboratory Manual. Cold Spring Harbor Laboratory Press, New York.

[13] Zhu, J., Zheng, A., Tan, F., et al. (2010) Characterisation and Expression of a Novel Holotype Crystal Protein Gene, cry56Aa1, from Bacillus thuringiensis Strain Ywc2-8. Biotechnology Letters, 32, 283-288. https://doi.org/10.1007/s10529-009-0147-6

[14] Ragni, A., Thiéry, I. and Delécluse, A. (1996) Characterization of Six Highly Mosquitocidal Bacillus thuringiensis Strains That Do Not Belong to H-14 Serotype. Current Microbiology, 32, 48-54. https://doi.org/10.1007/s002849900009

[15] Juárez-Pérez, V., Guerchicoff, A., Rubinstein, C., et al. (2002) Characterization of 
Cyt2Bc Toxin from Bacillus thuringiensis subsp. Medellin. Applied and Environmental Microbiology, 68, 1228-1231. https://doi.org/10.1128/AEM.68.3.1228-1231.2002

[16] Biasini, M., Bienert, S., Waterhouse, A., et al. (2014) SWISS-MODEL: Modelling Protein Tertiary and Quaternary Structure Using Evolutionary Information. Nucleic Acids Research, 42, 252-258. https://doi.org/10.1093/nar/gku340

[17] Laskowski, R.A., MacArthur, M.W., Moss, D.S., et al. (1993) PROCHECK: A Program to Check the Stereochemical Quality of Protein Structures. Journal of Applied Crystallography, 26, 283-291. https://doi.org/10.1107/S0021889892009944

[18] Robert, X. and Gouet, P. (2014) Deciphering Key Features in Protein Structures with the New ENDscript Server. Nucleic Acids Research, 42, 320-324. https://doi.org/10.1093/nar/gku316

[19] Pieper, U., Eswar, N., Davis, F.P., et al. (2006) MODBASE: A Database of Annotated Comparative Protein Structure Models and Associated Resources. Nucleic Acids Research, 34, 291-295. https://doi.org/10.1093/nar/gkj059

[20] Chothia, C. and Lesk, A.M. (1986) The Relation between the Divergence of Sequence and Structure in Proteins. The EMBO Journal, 5, 823.

https://doi.org/10.1002/j.1460-2075.1986.tb04288.x

[21] Wiederstein, M. and Sippl, M.J. (2007) ProSA-Web: Interactive Web Service for the Recognition of Errors in Three-Dimensional Structures of Proteins. Nucleic Acids Research, 35, 407-410. https://doi.org/10.1093/nar/gkm290

[22] Weng, Y.P., Lin, Y.P., Hsu, C.I., et al. (2004) Functional Domains of a Pore-Forming Cardiotoxic Protein, Volvatoxin A2. The Journal of Biological Chemistry, 279, 6805-6814. https://doi.org/10.1074/jbc.M308675200

[23] Promdonkoy, B. and Ellar, D.J. (2005) Structure-Function Relationships of a Membrane Pore Forming Toxin Revealed by Reversion Mutagenesis. Molecular Membrane Biology, 22, 327-337. https://doi.org/10.1080/09687860500166192

[24] Chang, C., Yu, Y.M., Dai, S.M., et al. (1993) High-Level cryIVD and cytA Gene Expression in Bacillus thuringiensis Does Not Require the 20-Kilodalton Protein, and the Coexpressed Gene Products Are Synergistic in Their Toxicity to Mosquitoes. Applied and Environmental Microbiology, 59, 815-821. https://doi.org/10.1128/AEM.59.3.815-821.1993

[25] Pérez, C., Fernandez, L.E., Sun, J., et al. (2005) Bacillus thuringiensis subsp. israelensis Cyt1Aa Synergizes Cry11Aa Toxin by Functioning as a Membrane-Bound Receptor. Proceedings of the National Academy of Sciences of the United States of America USA, 102, 18303-18308. https://doi.org/10.1073/pnas.0505494102

[26] Cantón, P.E., Reyes, E.Z., De Escudero, I.R., et al. (2011) Binding of Bacillus thuringiensis subsp. israelensis Cry4Ba to Cyt1Aa Has an Important Role in Synergism. Peptides, 32, 595-600. https://doi.org/10.1016/j.peptides.2010.06.005 